\title{
PRESCRIÇÃO DE QUELANTES DE FÓSFORO E CALCITRIOL PARA PACIENTES EM HEMODIÁLISE CRÔNICA
}

\author{
Maria Tereza Silveira Martins', luciana Ferreira da Silva ${ }^{2}$, Márcia Tereza Silva Martins ${ }^{3}$, Cácia Mendes Matos ${ }^{4}$, Nelson Almeida D'ávila Melo 5 , \\ Matheus Freitas Cardoso de Azevedo ${ }^{6}$, Iane Érica M. Travessa 7 , Maurício Kauark Amoedo ${ }^{8}$, Pedro Amoedo Fernandes ${ }^{9}$, Fernanda Conceição \\ Pereira Nogueira ${ }^{10}$, Gildete Barreto Lopes ${ }^{11}$, Antonio Alberto Lopes*12 \\ Trabalho realizado pelo Programa de Pós-graduação em Medicina e Saúde, Faculdade de Medicina, UFBA, Salvador, BA; na Clínica NEPHRON - \\ Barris, Salvador, BA; no Instituto de Nefrologia e Diálise (INED), Salvador, BA; na Clínica do Rim e Hipertensão Arterial (CLINIRIM), Salvador, BA; \\ no Núcleo de Epidemiologia Clínica do Hospital Universitário Professor Edgar Santos, Universidade Federal da Bahia (UFBA), Salvador, BA; e pelo \\ Departamento de Medicina, Faculdade de Medicina, UFBA, Salvador, BA.
}

\author{
*Correspondência: \\ Rua Marechal Floriano, 448 \\ - apt 1301 - Canela \\ CEP $40110-010$ \\ Salvador - BA \\ aaslopes@ufba.br
}

\begin{abstract}
RESUMO
OBjetıvo. Descrever a frequência de prescrição de quelantes de fósforo (QF) e calcitriol em pacientes sob hemodiálise (HD) crônica em Salvador, Brasil, e avaliar se o tratamento está de acordo com recomendações do Kidney Disease Outcomes Quality Initiative (K/DOQI).

Métodos. Corte transversal de dados da linha de base do Estudo Prospectivo do Prognóstico de Pacientes Tratados Cronicamente por Hemodiálise (PROHEMO). Foi realizada descrição da frequência de prescrição de QF e calcitriol conforme as concentrações de indicadores laboratoriais do metabolismo mineral, comparando com recomendações do K/DOQI.

Resultados. Sevelamer isoladamente (i.e., não combinado com outro QF) foi prescrito para 45,4\% dos pacientes, carbonato de cálcio ( $\mathrm{CaCO}$ ) isoladamente para 26,5\%, sevelamer combinado com $\mathrm{CaCO}$ para 2,1\% e acetato de cálcio para 5,2\%. Prescrição de QF foi observada para 53\% dos pacientes com fósforo $<3,5 \mathrm{mg} / \mathrm{dL}$ e $40 \%$ com fósforo $<3,0$ $\mathrm{mg} / \mathrm{dL}$. Em desacordo com K/DOQI, prescrição de calcitriol foi detectada para $19 \%$ dos pacientes com PTH $<150 \mathrm{pg} / \mathrm{mL}$ e ausência da prescrição para aproximadamente $35,4 \%$ com PTH $>300$ pg/dL combinado com fósforo menor ou igual a $5,5 \mathrm{mg} / \mathrm{dL}$, cálcio menor ou igual a $9,5 \mathrm{mg} / \mathrm{dL}$ e produto cálcio e fósforo (CaxP) $<55 \mathrm{mg} 2 / \mathrm{dL} 2$. Neste último grupo, 38\% tiveram prescrição de sevelamer sem outro QF.

Conclusão. Os resultados mostram um elevado percentual de prescrição de sevelamer em pacientes em HD de manutenção em uma cidade brasileira, apesar do alto custo deste medicamento e ausência de contraindicação para QF à base de cálcio. Os resultados em pacientes com PTH $<150 \mathrm{pg} / \mathrm{mL}$ e com PTH $>300 \mathrm{pg} / \mathrm{mL}$ combinado com determinadas concentrações de cálcio, fósforo e CaxP indicam também a necessidade de avaliar as práticas de uso de QF e calcitriol.
\end{abstract}

UnITERMOS: Hemodiálise. Metabolismo mineral. Quelante de fósforo. Vitamina D.

1. Mestre em Medicina e Saúde pela Universidade Federal da Bahia - Médica Nefrologista da Clínica NEPHRON. Salvador,BA, Brasil

2. Mestre em Alimentos e Nutrição - Nutricionista do Instituto de Nefrologia e Diálise; Professora Assistente da Universidade do Estado da Bahia; Aluna do Curso de Doutorado do Programa de Pós-Graduação em Medicina e Saúde da Faculdade de Medicina, UFBA, Salvador, BA, Brasil

3. Médica Nefrologista - Aluna do Curso de Mestrado do Programa de Pós-Graduação de Medicina e Saúde da Universidade Federal da Bahia. Médica Nefrologista da CLINIRIM, Salvador,BA

4. Mestre em Nefrologia - Diretora Médica do Instituto de Nefrologia e Diálise. Salvador, BA, Brasil

5. Médico Residente do Hospital Universitário Professor Edgard Santos, UFBA, Salvador, BA, Brasil

6. Médico Residente da Santa Casa de São Paulo. São Paulo,SP, Brasil

7. Estudante de Medicina - Bolsista de Iniciação Científica - Escola Bahiana de Medicina e Saúde Pública. Salvador,BA, Brasil

8. Médico Residente da Faculdade de Medicina de São José do Rio Preto. São José do Rio Preto,SP,Brasil

9. Estudante de Medicina - Bolsista de Iniciação Científica do PIBIC - Faculdade de Medicina da Bahia da Universidade Federal da Bahia. Salvador,BA,Brasil

10. Estudante de Medicina - Bolsista de Iniciação Científica do PIBIC - Faculdade de Medicina da Bahia da Universidade Federal da Bahia. Salvador, BA, Brasi

11. Mestre em Medicina e Saúde - Aluna do Curso de Doutorado do Programa de Pós-graduação em Medicina e Saúde da Universidade Federal da Bahia. Salvador, BA, Brasil

12. PhD em Ciência Epidemiológica pela Universidade de Michigan, Estados Unidos - Professor Associado I, Livre-Docente de Nefrologia da Faculdade de Medicina da Bahia da Universidade Federal da Bahia. Salvador,BA, Brasil 
PRESCRIÇÃo de QUELANTES DE FÓSFORO E CALCITRIOL PARA PACIENTES EM HEMODIÁLISE CRÔNICA

\section{INTRODUÇÃO}

O projeto das diretrizes do Kidney Disease Quality Initiative (K/DOQI) foi desenvolvido e tem sido divulgado amplamente pela National Kidney Foundation no intuito de que sejam adotadas práticas de diagnóstico, prevenção e tratamento das doenças renais baseadas nas melhores evidências dos trabalhos científicos'. Diretrizes foram especialmente desenvolvidas para a abordagem das alterações do metabolismo mineral2. De acordo com estas recomendações, pacientes em diálise devem manter as concentrações de cálcio sérico corrigido para a albumina entre 8,4-9,5 mg/dL, fósforo sérico entre 3,5-5,5 mg/ $\mathrm{dL}$, produto cálcio $x$ fósforo menor que $55 \mathrm{mg}^{2} / \mathrm{dL}^{2}$ e paratormônio (PTH) plasmático entre $150-300 \mathrm{pg} / \mathrm{mL}$. Para alcançar os alvos do controle do fósforo, o K/DOQI considera que tanto o sevelamer quanto os demais quelantes de fósforo podem ser prescritos inicialmente, considerando que são eficazes em manter o nível de fósforo dentro do recomendado em qualquer estágio da doença renal2. O K/DOQI, no entanto, recomenda que se evite o uso de quelante de fósforo à base de cálcio em pacientes com concentração sérica de cálcio, corrigido para a concentração de albumina, superior a $10,2 \mathrm{mg} / \mathrm{dL}$ ou com nível de PTH plasmático inferior a $150 \mathrm{pg} / \mathrm{mL}$ em duas avaliações consecutivas $^{2}$. As recomendações descritas nas diretrizes do K/DOQI para o metabolismo mineral visam reduzir as complicações, sobretudo os diversos tipos de doença óssea e a mortalidade precoce nos pacientes portadores de doença renal crônica ${ }^{3}$.

Em relação à prescrição de análogo de vitamina $\mathrm{D}$ para pacientes em diálise, o K/DOQI recomenda o seu uso quando a concentração sérica do PTH for superior a $300 \mathrm{pg} / \mathrm{mL}^{2}$. Caso ocorra aumento do cálcio, do fósforo e/ou do produto cálcio x fósforo para níveis acima do recomendado pelo $\mathrm{K} / \mathrm{DOQ}$ I durante o uso da vitamina $\mathrm{D}$, esta deverá ser reduzida ou mesmo interrompida². Caso a concentração de PTH se reduza para um limite inferior ou igual a $300 \mathrm{pg} / \mathrm{mL}$, recomenda-se a diminuição da dose ou a interrupção da prescrição do análogo da vitamina $D^{2}$.

O objetivo do presente estudo é descrever a frequência de prescrição dos diferentes tipos de quelantes de fósforo e do calcitriol (único análogo da vitamina D disponível para a população estudada) em pacientes sob hemodiálise crônica na cidade de Salvador. Os dados foram também analisados para verificar os percentuais de pacientes dentro e fora dos alvos recomendados pelo K/DOQI para cada indicador laboratorial do metabolismo mineral.

\section{Métodos}

Corte transversal de dados da linha de base do "Estudo Prospectivo do Prognóstico de Pacientes Tratados Cronicamente por Hemodiálise" (Estudo PROHEMO), desenvolvido em Salvador. Os dados analisados são de quatro clínicas participantes do estudo. 0 PROHEMO foi aprovado pelo Comitê de Ética em Pesquisa da Faculdade de Medicina da Universidade Federal da Bahia. Todos os pacientes assinaram o termo de consentimento livre e esclarecido para participação no estudo.

Foram estudados 747 pacientes adultos em HD crônica. Os pacientes que compõem a presente amostra foram admitidos no estudo no período de maio de 2005 a abril de 2007. Os dados foram obtidos através de questionário pré-definido e de informações sobre prescrição nos prontuários médicos. Os indicadores de adequação do metabolismo mineral (concentração sérica de cálcio, fósforo, PTH intacto e produto cálcio $\mathrm{x}$ fósforo) corresponderam ao valor laboratorial mais próximo à coleta dos dados clínicos e sociodemográficos, sendo que a rotina de exames dos serviços participantes inclui dosagem mensal de cálcio e fósforo e semestral de PTH. O PTH intacto foi dosado por eletroquimioluminescência (ROCHE). O cálcio foi corrigido para a concentração de albumina utilizando a seguinte fórmula: cálcio corrigido em $\mathrm{mg} / \mathrm{dL}=$ cálcio em mg/dL + (0,8 $\times(4$ - albumina em $\mathrm{g} / \mathrm{dL}))$.

Foram determinados para cada subgrupo, definido pelas concentrações dos indicadores laboratoriais do metabolismo mineral, os percentuais de pacientes com prescrição de carbonato de cálcio ou de sevelamer não combinado com outro quelante de fósforo ou com prescrição de calcitriol. Os percentuais de prescrição foram comparados com a conduta terapêutica recomendada pelo K/DOQI.

\section{Resultados}

A Tabela I descreve características gerais dos 747 pacientes participantes do estudo. A amostra foi constituída por 432 homens (57,9\%) e 315 mulheres (42,1\%). A média de idade dos pacientes era 48,5 $\pm 13,9$ anos (mediana $=48,3$ anos). Aproximadamente $50 \%$ dos pacientes estavam em tratamento dialítico há pelo menos 35 meses. Mulatos corresponderam a $61,6 \%$ da amostra, negros a $28,2 \%$ e brancos $8,7 \%$. As três causas mais frequentes de estágio final de doença renal (EFDR) identificadas pelos nefrologistas foram nefropatia hipertensiva (36\%), glomerulonefrite crônica $(27,2 \%)$ e nefropatia diabética (16,9\%). Diabetes mellitus como causa de EFDR ou comorbidade foi diagnosticada em 19,7\% (I47/747) dos pacientes. A média de albumina sérica foi $3,9 \pm 0,5 \mathrm{~g} / \mathrm{dL}$ (mediana $=4 \mathrm{~g} / \mathrm{dL}$ ) e de hemoglobina $10,1 \pm 1,7 \mathrm{~g} / \mathrm{dL}$ (mediana $=10,2$ ). A média de Kt $N$ foi de $1,5 \pm 0,2$ (mediana $=1,5)$.

Os percentuais de pacientes com concentrações de indicadores do metabolismo mineral dentro do recomendado pelo $\mathrm{K} / \mathrm{DOQ}$ foram 51,3\% para cálcio corrigido para albumina, 47,0\% para fósforo, 21,9\% para PTH e 68,9\% para produto cálcio e fósforo. Em torno de $13 \%$ dos pacientes apresentaram concentraç̃oes de cálcio corrigido abaixo do recomendado (i.e., $<8,4 \mathrm{mg} / \mathrm{dL}$ ) e 35,5\% acima do recomendado pelo $\mathrm{K} / \mathrm{DOQI}$ (i.e., $>9,5 \mathrm{mg} / \mathrm{dL}$ ). No que se refere ao $\mathrm{PTH}$, os percentuais de pacientes com concentrações abaixo (i.e., $<150 \mathrm{pg} / \mathrm{mL}$ ) e acima (i.e., $>300 \mathrm{pg} / \mathrm{ml}$ ) foram, respectivamente, $37,2 \%$ e 40,9\%. Aproximadamente $13,8 \%$ dos pacientes apresentaram concentrações de fósforo abaixo do recomendado (i.e., $<3,5 \mathrm{mg} / \mathrm{dL}$ ) e $7,8 \%$ concentrações abaixo de $3,0 \mathrm{mg} / \mathrm{dL}$.

A Tabela 2 mostra a frequência de prescrição de quelantes e de calcitriol entre os 747 pacientes. Foi observado que 79, I\% (59|/747) receberam prescrição de algum tipo de quelante de fósforo, sendo hidrocloreto de sevelamer o mais frequente prescrito (47,5\%). Carbonato de cálcio foi prescrito como quelante de fósforo para 28,6\% dos pacientes, sendo prescrito conjuntamente com sevelamer para 2, $1 \%$. Prescrição de acetato de cálcio foi observada para 5,2\% e de hidróxido de alumínio como quelante de fósforo para $0,7 \%$ dos pacientes.

A Tabela 3 mostra os percentuais de pacientes com prescrição de carbonato de cálcio, sevelamer não combinado a outro quelante de 


\begin{tabular}{|c|c|}
\hline \multicolumn{2}{|c|}{ Tabela I - Características gerais da amostra $(n=747)$} \\
\hline Característica & \\
\hline Idade em anos (média $\pm D P)$ & $48,5 \pm 13,9($ mediana $=48,3)$ \\
\hline $\begin{array}{l}\text { Grupos etários, \% (n) } \\
18-29 \\
30-49 \\
50-59 \\
60-69 \\
\geq 70\end{array}$ & $\begin{array}{c}10,3 \%(77) \\
44,0 \%(329) \\
24,0 \%(179) \\
\mid 4,9 \%(|| \mid) \\
6,8 \%(51)\end{array}$ \\
\hline $\begin{array}{l}\text { Sexo masculino, \% (n/N) } \\
\text { Raça, \%, (n) } \\
\text { Branca } \\
\text { Mulata } \\
\text { Negra } \\
\text { Outras }\end{array}$ & $\begin{array}{c}57,9 \%(4327747) \\
8,7 \%(65) \\
61,6 \%(460) \\
28,2 \%(211) \\
1,5 \%(11)\end{array}$ \\
\hline $\begin{array}{l}\text { Laboratório (média } \pm \text { DP /media } \\
\text { Albumina }(g / d) \text { (I) } \\
\text { Hemoglobina (g/d) }\end{array}$ & $\begin{array}{c}3,9 \pm 0,5(\text { mediana }=4,0) \\
\mid 0, I \pm 1,7(\text { mediana }=10,2)\end{array}$ \\
\hline $\begin{array}{l}\text { Nefropatia \% (n) } \\
\text { Hipertensiva } \\
\text { Glomerulonefrite } \\
\text { Diabética } \\
\text { Outras } \\
\text { Sem informação }\end{array}$ & $\begin{array}{c}36,0 \%(269) \\
27,2 \%(203) \\
16,9 \%(126) \\
19,0 \%(142) \\
0,9 \%(7)\end{array}$ \\
\hline Kt/V (média $\pm D P)$ & $1,5 \pm 0,2($ mediana $=1,5)$ \\
\hline Diabetes, \% (n/N) & $19,7 \%(147 / 747)$ \\
\hline Meses em diálise (média $\pm D P)$ & $52,7 \pm 53,9($ mediana $=35,4)$ \\
\hline
\end{tabular}

\begin{tabular}{lc}
\hline \multicolumn{2}{c}{ Tabela 2 - Frequência de prescrição de quelantes de fósforo e calcitrol } \\
\hline Quelante de fósforo & $\%(\mathbf{n} / \mathbf{N})$ \\
\hline Qualquer tipo de quelante & $79, I(59 \mid / 747)$ \\
Carbonato de cálcio & $28,6(214 / 747)$ \\
Carbonato de cálcio combinado com sevelamer & $2,1(16 / 747)$ \\
Sevelamer & $47,5(355 / 747)$ \\
Sevelamer sem carbonato de cácio & $45,4(339 / 747)$ \\
Acetato de cálcio & $5,2(39 / 747)$ \\
Hidróxido de alumínio & $0,7(5 / 747)$ \\
Calcitriol & $31,9(238 / 747)$ \\
\hline
\end{tabular}

fósforo e de calcitriol de acordo com concentrações de indicadores laboratoriais do metabolismo mineral. Na coluna direita da tabela é mostrada a conduta terapêutica esperada de acordo com as diretrizes $\mathrm{K} / \mathrm{DOQ}$. De acordo com as diretrizes $\mathrm{K} / \mathrm{DOQ}$ é esperado que pacientes com cálcio corrigido para albumina $>10,2 \mathrm{mg} / \mathrm{dL}$ (situação 2) não usem quelante de fósforo contendo cálcio e também calcitriol. Observou-se, no entanto, que carbonato de cálcio foi prescrito para 22,5\% e calcitriol para 23,4\% dos II I pacientes com cálcio corrigido $>10,2 \mathrm{mg} / \mathrm{dL}$. Dentre os 260 pacientes com PTH inferior a $150 \mathrm{pg} / \mathrm{mL}$ (situação 3), 32,3\% (84) receberam prescriçãao de carbonato de cálcio e 16,5\% (43) calcitriol, quando estas medicações deveriam ser evitadas de acordo com K/DOQI.
Em 103 pacientes com fósforo menor que 3,5 mg/dL (dados não mostrados na tabela) em que 0 esperado seria evitar quelantes de fósforo, 31,1\% tiveram prescrição de carbonato de cálcio e 16,5\% de sevelamer. Entre 58 pacientes com concentração de fósforo ainda mais baixa ( $<3,0 \mathrm{mg} / \mathrm{dL}$ ), 27,6\% tiveram prescrição de carbonato de cálcio e 6,9\% de sevelamer não combinado a outro quelante de fósforo (situação 5).

Dentre os 29 | pacientes com fósforo superior a 5,5mg/dL (situação 6), 66,3\% (193) receberam prescrição de sevelamer não combinado com outro quelante de fósforo e $23,7 \%$ receberam prescrição de calcitriol. Entre os 222 pacientes com produto cálcio x fósforo $\geq 55 \mathrm{mg}^{2} /$ $L^{2}, 19,8 \%$ tiveram prescrição de calcitriol e $14 \%$ de carbonato de cálcio (situação 7).

A Tabela 3 mostra também os percentuais de prescrição de quelantes de fósforo e calcitriol de acordo com combinações dos indicadores do metabolismo mineral. Dentre os 41 pacientes com a combinação mostrada na situação 8, 58,5\% (24/4I) tiveram prescrição de sevelamer e 29,3\% (I2/4I) de carbonato de cálcio e, contrariando as diretrizes $\mathrm{K} / \mathrm{DOQ}$, 43,9\% fizeram uso de calcitriol.

As situações 9 e 10 representam os pacientes com PTH $>300$ combinados com diferentes concentrações dos demais indicadores laboratoriais do metabolismo mineral. De acordo com as recomendações do K/DOQI, na situação 9 (cálcio >9,5 ou fósforo $>5,5$ ou CaxP $\geq 55$ combinado com PTH>300) o uso de calcitriol deve ser evitado e na situação 10 (cálcio $\leq 9,5$ e fósforo $\geq 5,5$ e CaxP $<55$ e PTH $>300$ ) está indicado o uso de calcitriol. Conforme mostrado na Tabela 3, 34,3\% dos pacientes tiveram prescrição de calcitriol na situação 9 e $35,4 \%$ dos pacientes não tiveram prescrição de calcitriol na situação 10.

\section{Discussão}

De acordo com os resultados do início da coleta de dados do PROHEMO, um percentual elevado de pacientes não havia alcançado os alvos recomendados pelo K/DOQI para os indicadores do metabolismo mineral, i.e., cálcio corrigido para albumina, fósforo, PTH e produto cálcio x fósforo. Foi também observada uma discordância com - K/DOQI na prescrição de quelantes de fósforo e de calcitriol. Observou-se, por exemplo, prescrição de quelantes de fósforo para mais de um terço dos pacientes com fósforo sérico inferior a $3 \mathrm{mg} / \mathrm{dL}$, portanto muito abaixo do alvo recomendado pelo K/DOQI. Tal fato se repetiu na avaliação da prescrição de calcitriol de acordo com as orientações do K/DOQI. Foi observada prescrição de calcitriol para quase um quinto dos pacientes com $\mathrm{PTH}<150 \mathrm{pg} / \mathrm{mL}$, possivelmente contribuindo para aumentar a possibilidade de doença óssea adinâmica. Por outro lado, não se detectou prescrição de calcitriol para mais de um terço dos pacientes com PTH $>300 \mathrm{pg} / \mathrm{dL}$, que pode estar associada a uma maior prevalência de hiperparatireoidismo secundário ${ }^{4-56}$.

Em relação à prescrição de quelantes de fósforo, quase metade dos pacientes tinha prescrição de sevelamer. Tal fato chamou atenção, pois além do perfil dos indicadores laboratoriais do metabolismo mineral e da presença de calcificação vascular, custo é outro fator a ser considerado na decisão entre usar sevelamer ou outro quelante de fósforo ${ }^{7}$. É importante notar que, no nosso meio, o custo do sevelamer é aproximadamente 60 vezes superior ao do carbonato de cálcio e 20 vezes superior ao acetato de cálcio 8.9 . 


\begin{tabular}{|c|c|c|c|c|c|c|}
\hline \multirow[b]{2}{*}{ Situação } & \multirow[b]{2}{*}{$\begin{array}{l}\text { Indicadores do } \\
\text { metabolismo mineral }\end{array}$} & \multirow[b]{2}{*}{ N } & \multicolumn{3}{|c|}{$\%$ Observado no Estudo } & \multirow[b]{2}{*}{$\begin{array}{l}\text { Esperado de acordo } \\
\text { com K/DOQI** }\end{array}$} \\
\hline & & & $\mathrm{CaCO}_{3}$ & $\begin{array}{l}\text { Sevelamer } \\
\text { isolado }\end{array}$ & Calcitriol & \\
\hline 1 & $\mathrm{Ca}<8,4$ & 94 & 35,1 & 38,3 & 37,2 & $\begin{array}{l}\text { Sem contraindicação para } \\
\text { quelante de cálcio se CaxP }<55\end{array}$ \\
\hline 2 & $\mathrm{Ca}>10,2$ & 111 & 22,5 & 53,2 & 23,4 & $\begin{array}{l}\text { Evitar quelante a base } \\
\text { de cálcio e calcitriol }\end{array}$ \\
\hline 3 & PTH $<150$ & 260 & 32,3 & 36,5 & 16,5 & $\begin{array}{l}\text { Evitar quelante a base de cálcio } \\
\text { e não prescrever calcitriol }\end{array}$ \\
\hline 4 & PTH $>300$ & 286 & 34,2 & 51,1 & 58,3 & $\begin{array}{l}\text { Uso de calcitriol na ausência } \\
\text { de CaxP elevado }\end{array}$ \\
\hline 5 & $P<3,0$ & 58 & 27,6 & 6,9 & 31,0 & Evitar quelantes de fósforo \\
\hline 6 & $P>5,5$ & 291 & 19,2 & 66,3 & 23,7 & Não prescrever calcitriol \\
\hline 7 & CaxP $>55$ & 222 & 14,0 & 71,6 & 19,8 & $\begin{array}{l}\text { Não prescrever calcitriol e } \\
\text { quelante a base de cácio }\end{array}$ \\
\hline 8 & $\begin{array}{c}\text { Ca } \leq 9,5 \text { e } P>5,5 \text { e } \\
\text { PTH }>150 \text { e CaxP }<55\end{array}$ & 41 & 29,3 & 58,5 & 43,9 & $\begin{array}{l}\text { Quelante a base de cácio ou sevelamer. } \\
\text { Se P>5,5 associar quelantes. Evitar calcitriol }\end{array}$ \\
\hline 9 & $\begin{array}{c}\mathrm{Ca}>9,5 \text { ou } \mathrm{P}>5,5 \text { ou } \\
\mathrm{CaxP} \geq 55 \text { com } \mathrm{PTH}>300\end{array}$ & 213 & 21,1 & 63,8 & 34,3 & Evitar calcitriol \\
\hline 10 & $\begin{array}{c}\mathrm{Ca} \leq 9,5 \text { e } \mathrm{P} \leq 5,5 \text { e } \\
\mathrm{CaxP}<55 \text { e PTH }>300\end{array}$ & 96 & 36,5 & 37,5 & 64,6 & Indicado o uso de calcitriol \\
\hline
\end{tabular}

* Indicadores laboratoriais representados nas seguintes unidades de medida: cálcio ( $\mathrm{Ca}$ e em mg/dL; fósforo (P) em mg/dL; produto cálcio x fósforo (CaxP) em mg $\mathrm{mg}^{2} / \mathrm{dL}^{2}$ e PTH em pg/mL; cálcio foi corrigido para a concentração de albumina.

** $\mathrm{K} / \mathrm{DOQI}=$ Kidney Disease Outcomes Quality Initiative

O K/DOQI sugere que em situações de persistência de hiperfosfatemia, apesar do uso de um quelante de fósforo, pode-se associar um segundo quelante ${ }^{2}$. É possível que o uso judicioso de associações de menores doses de sevelamer e de quelantes à base de cálcio possa melhorar o controle do fósforo sérico e ao mesmo tempo reduzir os custos do tratamento ${ }^{10}$. No nosso estudo, no entanto, observamos que somente um percentual muito pequeno (aproximadamente $2,1 \%$ ) de pacientes recebeu prescrição de sevelamer combinado com outro quelante.

No presente estudo observou-se que sevelamer foi o quelante de fósforo prescrito para aproximadamente $70 \%$ dos pacientes com fósforo superior a 5,5 mg/dL. Neste subgrupo de pacientes, sevelamer foi usado em lugar de quelantes contendo cálcio, mesmo em situações de hipocalcemia. O fato de não existirem evidências suficientes mostrando benefício na redução de mortalidade, hospitalização ou melhora da qualidade de vida associadas ao uso do hidrocloreto de sevelamer apoia a necessidade da reavaliação da prescrição dos quelantes de fósforo observada no presente estudo".

Tonelli et al. realizaram uma meta-análise que incluiu dez ensaios clínicos randomizados totalizando 3.000 pacientes em diálise que utilizaram sevelamer ou um dos quelantes à base de cálcio". Não foi observada diferença na qualidade de vida, taxa de mortalidade por todas as causas, mortalidade cardiovascular, efeitos adversos ou hospitalizações entre pacientes tratados com sevelamer e quelantes à base de cálcio".

O presente estudo apresenta informações que podem ajudar a melhorar o controle do metabolismo mineral em pacientes em hemodiálise crônica no nosso meio. Objetivando o uso adequado das informações, no entanto, é importante considerar potenciais limitações metodológicas. Como a análise se baseou em um corte transversal não foi possível avaliar modificações na prescrição bem como nas concentrações dos indicadores laboratoriais do metabolismo mineral ao longo da evolução dos pacientes. Outra limitação deste estudo foi a falta de informação sobre a presença de calcificações metastáticas, que podem justificar a prescrição de sevelamer em pacientes sem contraindicações ao uso de quelante à base de cálcio. O fato da dosagem de PTH ser semestral pode ter também contribuído para que um percentual elevado de pacientes esteja com prescrição de calcitriol em desacordo com recomendações do $\mathrm{K} / \mathrm{DOQ}$.

Em conclusão, os dados do presente estudo sugerem que um percentual considerável de pacientes em hemodiálise em Salvador tem prescrição de quelante de fósforo e calcitriol que diferem do que tem sido recomendado pelo $\mathrm{K} / \mathrm{DOQ}$. Uma compreensão dos fatores relacionados a esta inadequação e uma visão crítica das recomendações do K/DOQI deverão permitir melhor controle das alterações do metabolismo mineral e redução de custos desnecessários em nosso meio. 
MARTINS MTS ET AL.

\section{Agradecimentos}

Os autores agradecem aos diretores e equipes das clínicas de diálise participantes do PROHEMO e a Sociedade Brasileira de Nefrologia - Regional da Bahia pela colaboração para o desenvolvimento do estudo. O PROHEMO conta também com apoio do CNPq referente a recursos para custeio/capital (processo 484743/2006-6), bolsas de iniciação cientíica do Programa Institucional de Bolsas de Iniciação Cientifica (PIBIC) e bolsa de produtividade em pesquisa (processo 308068/2006-8).

Conflito de interesse: não há

\section{SUMMARY}

PRESCRIPTION OF PHOSPHORUS BINDERS AND CALCITRIOL FOR CHRONIC HEMODIALYSIS PATIENTS

OBIECTNE. To describe the frequency of prescription of phosphate binders $(P B)$ and calcitriol for patients on chronic hemodialysis in Salvador, Brazil, and to assess whether treatment is in agreement with recommendations of the Kidney Disease Outcomes Quality Initiative (K/DOQI).

MetHOdS. Cross section of baseline data of the PROHEMO study. The frequency of prescription of $P B$ and calcitriol according to laboratory indicators of mineral metabolism was compared with K/DOQI recommendations.

RESULTS. Sevelamer alone (i.e., not combined with other PB) was prescribed for $45.4 \%$ of patients, calcium carbonate ( $\mathrm{CaCO}$ ) alone for $26.5 \%$, sevelamer combined with $\mathrm{CaCO} 3$ for $2.1 \%$ and calcium acetate for $5.2 \%$. Prescription of PB was noted in $53 \%$ of the patients with phosphorus $<3.5 \mathrm{mg} / \mathrm{dL}$ and $40 \%$ with phosphorus $<3.0 \mathrm{mg} / \mathrm{dL}$. In disagreement with $\mathrm{K} / \mathrm{DOQ}$, prescription of calcitriol was found in $19 \%$ of patients with PTH $<150 \mathrm{pg} / \mathrm{mL}$ and prescription was absent for approximately $35.4 \%$ with PTH $>300 \mathrm{pg} / \mathrm{dL}$ combined with phosphorus equal or less than $5.5 \mathrm{mg} / \mathrm{dL}$, calcium equal or less than 9.5 $\mathrm{mg} / \mathrm{dL}$ and calcium $x$ phosphorus product $($ CaxP) $<55 \mathrm{mg} 2 / \mathrm{dL} 2$. For this latter group 38\% had a prescription of sevelamer without other phosphate binders.

CONCLUSION. Results show a large percentage of prescriptions of sevelamer among patients on maintenance hemodialysis in a Brazilian city, despite the high cost of the medication and absence of contraindications for $P B$ with calcium salts. Results in patients with $P T H<150 \mathrm{pg} / \mathrm{mL}$ and with PTH $>300 \mathrm{pg} / \mathrm{mL}$ combined with certain concentrations of calcium, phosphate and CaxP also indicate the need to evaluate practices for use of phosphate binders and calcitriol. [Rev Assoc Med Bras 2009; 55(I): 70-4]

KeY wORDS: Hemodialysis. Mineral metabolism. Phosphate binder. Vitamin D.

\section{REFERÊNCIAS}

I. The National Kidney Foundation Kidney Disease Outcomes Quality Initiative. [cited 2007 oct 13].Available from: http://www.kidney.org/ professionals/KDOQI.

2. National Kidney Foundation. K/DOQI clinical practice guidelines for bone metabolism and disease in chronic kidney disease. Am J Kidney Dis. 2003;42(Suppl):SI-20I.

3. Young EW, Akiba T, Albert JM, McCarthy JT, Kerr PG, Mendelssohn DC, et al. Magnitude and impact of abnormal mineral metabolism in hemodialysis patients in the Dialysis Outcomes and Practice Patterns Study (DOPPS). Am J Kidney Dis. 2004:44:34-8.

4. Slatopolsky E, Brown A, Dusso A. Pathogenesis of secondary hyperparathyroidism. Kidney Int Suppl. 1999;73:SI4-9.

5. Martin KJ, Olgaard K, Coburn JW, Coen GM, Fukagawa M, Langman C, et al. Diagnosis, assessment, and treatment of bone turnover abnormalities in renal osteodystrophy. Am J Kidney Dis. 2004:43:558-65.

6. Goodman WG, Ramirez JA, Belin TR, Chon Y, Gales B, Segre GV, et al. Development of adynamic bone in patients with secondary hyperparathyroidism after intermittent calcitriol therapy. Kidney Int 1994:46:1160-6

7. Palmer SC, Craig JC, Strippoli GF. Sevelamer: a promising but unproven drug. Nephrol Dial Transplant. 2007;22:2742-5.

8. Guia Farmacêutico Brasíndice [online]. [citado out 2007]. São Paulo: Andrei Publicações Médicas; 2007. Disponível em: http:// www.brasindice. com.br/conheca.shtml.

9. Quimibras Indústrias Químicas S/A. [online]. Rio de Janeiro. Especificações Reagen. Reagen lista de especificações por produtos. [citado 22 jan 2008]. Disponível em: http://www.quimibras.com.br/ homepage/especificacoes/espec Reagen.html. I0. Iwasaki Y, Takami $H$, Tani M, Yamaguchi Y, Goto $\bar{H}$, Goto $Y$, et al. Efficacy of combined sevelamer and calcium carbonate therapy for hyperphosphatemia in Japanese hemodialysis patients. Ther Apher Dial. 2005;9:347-5I .

I I. Tonelli M, Wiebe N, Culleton B, Lee H, Klarenbach S, Shrive F, et al. Systematic review of the clinical efficacy and safety of sevelamer in dialysis patients. Nephrol Dial Transplant. 2007;22:2856-66.

Artigo recebido: 18/02/08

Aceito para publicação: 17/05/08 